\title{
MODULARITY OF THE LATTICE OF CONGRUENCES OF A REGULAR $\omega$-SEMIGROUP*
}

\author{
by C. BONZINI and A. CHERUBINI
}

(Received 12th January 1989)

Dedicated to Professor C. Tibiletti Marchionna on her 70th birthday

\begin{abstract}
In this paper a characterization of the regular $\omega$-semigroups whose congruence lattice is modular is given. The characterization obtained for such semigroups generalizes the one given by Munn for bisimple $\omega$-semigroups and completes a result of Baird dealing with the modularity of the sublattice of the congruence lattice of a simple regular $\omega$-semigroup consisting of congruences which are either idempotent separating or group congruences.
\end{abstract}

1980 Mathematics subject classification (1985 Revision): 20M10

\section{Introduction}

A regular $\omega$-semigroup $S$ is a regular semigroup whose set of idempotents $E(S)$, or shortly $E$, forms an $\omega$-chain

$$
e_{0}>e_{1}>\cdots>e_{n}>\cdots
$$

under the natural order defined on $E$ by the rule $e \geqq f$ if and only if $e f=f=f e$. The congruences on this interesting class of inverse semigroups have been described (see, e.g., [2] and [9]), but this description has been not much used for the investigation of the congruence lattice $L(S)$.

As regards the modularity of $L(S)$, Munn [8] characterized bisimple $\omega$-semigroups whose lattice of congruences is modular, observing that the congruences on $S$ reduce to idempotent separating and group congruences. Baird [1] studied simple $\omega$-semigroups and gave a necessary and sufficient condition for the sublattice of $L(S)$ consisting of those congruences which are either idempotent separating or group congruences to be a modular lattice.

This paper completes the previous results giving the characterization of regular $\omega$ semigroups whose congruences lattice is modular.

As usual $\sigma$ denotes the least group congruence, $H$ and $D$ the Green's relations, $t$ the identity congruence on $S$ and $N$ the set of non-negative integers.

The undefined terminology and notation can be found in [10].

\section{Modularity of the lattice of congruences on simple regular $\omega$-semigroups}

This section deals with the characterization of simple regular $\omega$-semigroups whose

*Work supported by M.P.I. 
lattice of congruence is modular. (A lattice $L$ is modular if for every $a, b, c \in L$ with $a \leqq b, \quad a \cup c=b \cup c$ and $a \cap c=b \cap c$ imply $a=b$, or equivalently if we have $(a \cup c) \cap b=a \cup(c \cap b))$. Two authors, Kocin [5] and Munn [7], gave structure theorems for simple regular $\omega$-semigroups. The one given by Munn is the following:

Theorem A. Let $d$ be a positive integer and let $\left\{G_{i} \mid i=0, \ldots, d-1\right\}$ be a set of $d$ pairwise disjoint groups. Let $\gamma_{d-i}$ be a homomorphism of $G_{d-1}$ into $G_{0}$ and if $d>1$, let $\gamma_{i}$ be a homomorphism of $G_{i}$ into $G_{i+1}(i=0, \ldots, d-2)$. For every $n \in \mathbb{N}$ let $\gamma_{n}=\gamma_{n(\bmod d)}$ $(n(\bmod d))$ denote the integer equivalent to $n$ modulo $d$, belonging to $\mathbb{N}$ and less than $d)$. For $m, n \in \mathbb{N}$ and $m<n$ write

$$
\alpha_{m, n}=\gamma_{m} \gamma_{m+1} \ldots \gamma_{n-1}
$$

and for all $n \in \mathbb{N}$ let $\alpha_{n, n}$ denote the identity automorphism of $G_{n(\bmod d)}$.

Let $S$ be the set of the ordered triples $\left(m, a_{i}, n\right)$, where $m, n \in \mathbb{N}, 0 \leqq i \leqq d-1$ and $a_{i} \in G_{i}$. Define a multiplication in $S$ by the rule that

$$
\left(m, a_{i}, n\right)\left(p, b_{j}, q\right)=\left(m-n+t,\left(a_{i} \alpha_{u, w}\right)\left(b_{j} \alpha_{v, w}\right), q-p+t\right)
$$

where $t=\max \{n, p\}, u=n d+i, v=p d+j$ and $w=\max \{u, v\}$. (The multiplication in $S$ can be defined equivalently by the rule $\left(m, a_{i}, n\right)\left(p, b_{j}, q\right)=\left(m+p-r,\left(a_{i} \alpha_{u, w}\right)\left(b_{j} \alpha_{v, w}\right), n+q-r\right)$ where $r=\min \{n, p\}, u=n d+i, v=p d+j$ and $w=\max \{u, v\})$. Denote the so formed groupoid by $S\left(d, G_{i}, \gamma_{i}\right)$. Then $S\left(d, G_{i}, \gamma_{i}\right)$ is a simple regular $\omega$-semigroup with exactly $d$ $D$-classes and any simple regular $\omega$-semigroup is isomorphic to a semigroup $S\left(d, G_{i}, \gamma_{i}\right)$. For $n \in \mathbb{N}$ and $i=0, \ldots, d-1$ write $e_{i}^{n}=\left(n, e_{i}, n\right)$, where $e_{i}$ is the identity of the group $G_{i}$. The elements $e_{i}^{n}$ are the idempotents of $S\left(d, G_{i}, \gamma_{i}\right)$ and we have

$$
e_{0}^{0}>e_{1}^{0}>\cdots>e_{d-1}^{0}>e_{0}^{1}>\cdots>e_{d-1}^{1}>e_{0}^{2}>\cdots
$$

In the remainder of the paper the endomorphism $\alpha_{i, i+d}$ of $G_{i}$ will be indicated by $\alpha_{i}$. Moreover, in view of the above theorem, we will denote a simple regular $\omega$-semigroup by $S\left(d, G_{i}, \gamma_{i}\right)$.

Definition 1.1. (see $[2,2$ and 3], $[1,2])$. Let $S=S\left(d, G_{i}, \gamma_{i}\right)$. A congruence $\mu$ on the set $E$ of idempotents is called uniform if $\left(e_{i}^{n}, e_{j}^{m}\right) \in \mu$ implies that

$$
\left(e_{i}^{n+p}, e_{j}^{m+p}\right) \in \mu \text { for all integers } p \geqq-\min \{m, n\} .
$$

Put $G=G_{0} \times G_{1} \times \cdots \times G_{d-1}$, the cartesian product of $G_{i}, i=0,1, \ldots, d-1$. A subset $A$ of $G$ will be called $\gamma$-admissible if

(i) $A=A_{0} \times \cdots \times A_{d-1}$, for some $A_{i} \subseteq G_{i}, i=0,1, \ldots, d-1$.

(ii) $A_{i} \unlhd G_{i}$, for $i=0,1, \ldots, d-1$.

(iii) $A_{d-1} \gamma_{d-1} \subseteq A_{0}$ and $A_{i} \gamma_{i} \subseteq A_{i+1}$, for $i=0,1, \ldots, d-2$. 
A subset $A$ of $G$ will be called normal if it satisfies (i) and (ii) above. If $A=$ $A_{0} \times \cdots \times A_{d-1}$ and $B=B_{0} \times \cdots \times B_{d-1}$ are normal subsets of $G$ we define $A \cdot B=$ $A_{0} B_{0} \times \cdots \times A_{d-1} B_{d-1} \cdot A \cdot B$ is a normal subset and, if $A$ and $B$ are $\gamma$-admissible, $A \cdot B$ is $\gamma$-admissible.

Let $\mu$ be a uniform congruence on $E(S)$ and $A$ a $\gamma$-admissible subset of $G$. Put

$\mu-\operatorname{rad} A_{i}=\left\{a_{i} \in G_{i} \mid a_{i} \alpha_{n d+i, m d+j} \in A_{j}\right.$ for some $n, m, j$ such that $\left(e_{i}^{n}, e_{j}^{m}\right) \in \mu$ and $\left.e_{j}^{m} \leqq e_{i}^{n}\right\}$ and

$$
\mu-\operatorname{rad} A=\mu-\operatorname{rad} A_{0} \times \cdots \times \mu-\operatorname{rad} A_{d-1} .
$$

If $\mu-\operatorname{rad} A=A, \mu$ and $A$ are called linked.

Remark 1.2. (see [2], Lemma 3.1 and Lemma 3.2]) Let $\mu$ and $\mu^{\prime}$ be uniform congruences of $E(S)$ and let $A$ and $A^{\prime}$ be $\gamma$-admissible subsets of $G$. Then

(i) $\mu$-rad $A$ is a $\gamma$-admissible subset of $G$ and $A \subseteq \mu$-rad $A$,

(ii) $A \subseteq A^{\prime}$ implies $\mu-\operatorname{rad} A \subseteq \mu-\operatorname{rad} A^{\prime}$,

(iii) $\mu \leqq \mu^{\prime}$ implies $\mu-\operatorname{rad} A \subseteq \mu^{\prime}-\operatorname{rad} A$,

(iv) $\mu-\operatorname{rad} A=\mu-\operatorname{rad}(\mu-\operatorname{rad} A)$.

Hence, if we denote by 1 the $\gamma$-admissible subset $\left\{e_{0}\right\} \times \cdots \times\left\{e_{d-1}\right\}$ of $G$, it follows that

(v) $\mu-\operatorname{rad} 1 \subseteq \mu-\operatorname{rad} A$,

(vi) $A \cdot \mu-\operatorname{rad} 1 \subseteq \mu-\operatorname{rad} A$.

Theorem B. (See [2], Theorems 4.2, 5.1, 5.2, 5.3). Let $S=S\left(d, G_{i}, \gamma_{i}\right)$, let $\mu$ be a uniform congruence on $E$, let $A$ be a $\gamma$-admissible subset of $G$, and suppose that $\mu$ and $A$ are linked. Then

$$
\begin{aligned}
\tau= & \left\{\left(\left(m, a_{i}, n\right),\left(p, b_{j}, q\right)\right) \in S \times S \mid\left(a_{i} \alpha_{u, w}\right)\left(b_{j}^{-1} \alpha_{v, w}\right) \in A_{w}(\bmod d),\right. \\
& \text { where } \left.u=n d+i, v=q d+j, w=\max \{u, v\} ; m-n=p-q ;\left(e_{i}^{m}, e_{j}^{p}\right) \in \mu\right\}
\end{aligned}
$$

is a congruence on $S$ contained in $[1, \sigma \vee H]$ such that $\operatorname{tr} \tau=\mu$ and $A^{t}=A$, where $A=A_{0}^{\tau} \times \cdots \times A_{d-1}^{\tau}$ and $A_{i}^{\tau}=\left\{a_{i} \in G_{i} \mid\left(0, a_{i}, 0\right) \tau e_{i}^{0}\right\}, i=0, \ldots, d-1$.

Conversely, let $\tau$ be a congruence on $S=S\left(d, G_{i}, \gamma_{i}\right)$ contained in $[l, \sigma \vee H]$, then $\tau$ is of the above form with $\mu=\operatorname{tr} \tau$ and $A=A^{\tau}$.

Moreover, let $\rho, \lambda$ be congruences on $S=S\left(d, G_{i}, \gamma_{i}\right)$ contained in $[1, \sigma \vee H]$. Then

(i) $\rho \leqq \lambda$ if and only if $\operatorname{tr} \rho \leqq \operatorname{tr} \lambda$ and $A^{\rho} \subseteq A^{\lambda}$,

(ii) $\operatorname{tr}(\rho \vee \lambda)=\operatorname{tr} \rho \vee \operatorname{tr} \lambda, \operatorname{tr}(\rho \wedge \lambda)=\operatorname{tr} \rho \wedge \operatorname{tr} \lambda$,

(iii) $A^{\rho \vee \lambda}=(\operatorname{tr} \rho \vee \operatorname{tr} \lambda)-\operatorname{rad} A^{\rho} \cdot A^{\lambda}, A^{\rho \wedge \lambda}=A^{\rho} \cap A^{\lambda}$.

Lemma 1.3. Let $S=S\left(d, G_{i}, \gamma_{i}\right)$. Let $\mu$ and $v$ be uniform congruences on $E(S)$ and let $A$ 
be a $\gamma$-admissible subset of $G$. If $A$ is linked with both $\mu$ and $v$, then it is also linked with $\mu \vee v$.

Proof. We must prove that $(\mu \vee v)$-rad $A=A$. Since $A=A_{0} \times \cdots \times A_{d-1}$, first we prove that $(\mu \vee v)-\operatorname{rad} A_{i}=A_{i}$ for every $i=0, \ldots, d-1$. Let $g_{i} \in(\mu \vee v)$-rad $A_{i}$, then $g_{i} \in G_{i}, \quad g_{i} \alpha_{n d+i, m d+j} \in A_{j}$ with $n, m \in \mathbb{N}, j=0, \ldots, d-1$ such that $e_{j}^{m} \leqq e_{i}^{n}, e_{j}^{m}(\mu \vee v) e_{i}^{n}$. Hence there exist $e_{i}^{n}=e_{i_{1}}^{n_{1}}, \ldots, e_{i_{r}}^{n_{r}}, \ldots, e_{i_{k}}^{n_{k}}=e_{j}^{m} \in E \quad\left(0 \leqq i_{r} \leqq d-1, n \leqq n_{r} \leqq m\right)$ such that $e_{i_{1}}^{n_{1}} \geqq \cdots \geqq e_{i_{r}}^{n_{r}} \geqq \cdots \geqq e_{i_{k}}^{n_{k}} ; e_{i_{1}}^{n_{1}} \mu e_{i_{2}}^{n_{2}}, e_{i_{2}}^{n_{2}} \vee e_{i_{3}}^{n_{3}}, \ldots, e_{i_{k-1}}^{n_{k-1}} \vee e_{i_{k}}^{n_{k}}$. Hence we have $g_{i} \alpha_{n d+i, m d+j}=$ $\left(g_{i} \alpha_{n d+i, n_{k}-1 d+i_{k-1}}\right) \alpha_{n_{k}-1 d+i_{k-1}, m d+j} \in A_{j}$. Since we have $g_{i} \alpha_{n d+i, n_{k-1} d+i_{k-1}} \in G_{i_{k-1}}, e_{i_{k-1}}^{n_{k-1}} v e_{j}^{m}$, $e_{i_{k-1}}^{n_{k-1}} \geqq e_{j}^{m}$, it follows that $g_{i} \alpha_{n d+i, n_{k-1} d+i_{k-1}} \in v-\operatorname{rad} A_{i_{k-1}}$. Hence, since $A$ is linked with $v$, we have $v-\operatorname{rad} A=A$ whence $v-\operatorname{rad} A_{i_{k-1}}=A_{i_{k-1}}$, thus $g_{i} \alpha_{n d+i, n_{k-1} d+i_{k-1}} \in A_{i_{k-1}}$. Repeating the same argument, since we have also $A=\mu-\operatorname{rad} A$, we obtain $g_{i} \in A_{i}$. It follows that $(\mu \vee v)-\operatorname{rad} A_{i}=A_{i}$, hence $(\mu \vee v)-\operatorname{rad} A=A$.

Lemma 1.4. Let $S=S\left(d, G_{i}, \gamma_{i}\right)$. If $L(S)$ is modular, then the following condition holds

$$
A \cdot \mu-\operatorname{rad} 1=\mu-\operatorname{rad} A
$$

for every $\gamma$-admissible subset $A$ of $G$ and for every uniform congruence $\mu$ on $E(S)$.

Proof. Let $A$ be a $\gamma$-admissible subset of $G$ and $\mu$ a uniform congruence on $E(S)$. We remark that every $\gamma$-admissible subset is linked with the identity on $E(S)$ which is obviously uniform, moreover $\mu$-rad 1 is a $\gamma$-admissible subset and it is linked with the uniform congruence $\mu$.

Hence, by Theorem $\mathrm{B}$, there exist in $L(S)$ three congruence $\rho, \lambda, \tau$ such that $\rho, \lambda$ are idempotent separating with $A^{\rho}=A \cdot \mu-\operatorname{rad} 1, A^{\lambda}=\mu-\operatorname{rad} A$, and $\tau$ has trace $\mu$ with $A^{z}=\mu$-rad 1. Thus, we obtain, by Theorem B (i) and by Remark 1.2 (vi)

$$
\rho \leqq \lambda
$$

and by Theorem $\mathbf{B}$ (ii)

$$
\operatorname{tr}(\rho \wedge \tau)=\operatorname{tr}(\lambda \wedge \tau)=l, \quad \operatorname{tr}(\rho \vee \tau)=\operatorname{tr}(\lambda \vee \tau)
$$

Moreover by Theorem B (iii), and by Remark 1.2 (ii), (iv), (v), (vi) $A^{i \vee \tau}=$ $\mu-\operatorname{rad}(\mu-\operatorname{rad} A \cdot \mu-\operatorname{rad} 1)=\mu-\operatorname{rad}(\mu-\operatorname{rad} A)=\mu-\operatorname{rad} A, \quad A^{\rho \vee \tau}=\mu-\operatorname{rad}(A \cdot \mu-\operatorname{rad} 1 \cdot \mu-\operatorname{rad} 1)=$ $\mu-\operatorname{rad}(A \cdot \mu-\operatorname{rad} 1)=\mu-\operatorname{rad} A$, thus

$$
A^{\rho \vee \tau}=A^{\lambda \vee \tau}=\mu-\operatorname{rad} A, \quad A^{\rho \wedge \tau}=A^{\lambda \wedge \tau}=\mu-\operatorname{rad} 1 .
$$

Since $L(S)$ is modular, (1), (2), (3) imply $\rho=\lambda$, whence, by Theorem 2 , condition (*) holds.

Lemma 1.5. Let $S=S\left(d, G_{i}, \gamma_{i}\right)$. If condition (*) holds, then 


$$
\mu-\operatorname{rad} 1 \cdot v-\operatorname{rad} 1=(\mu \vee v)-\operatorname{rad} 1
$$

for every $\mu, v$ uniform congruences on $E(S)$.

Proof. Putting $A=v$-rad 1, from condition (*) it follows that $v$-rad $1 \cdot \mu$-rad $1=$ $\mu-\operatorname{rad}(v-\operatorname{rad} 1)$. Hence, by Remark $1.2($ iv $), \mu-\operatorname{rad}(v-\operatorname{rad} 1 \cdot \mu-\operatorname{rad} 1)=\mu-\operatorname{rad}(\mu-\operatorname{rad}(v-\operatorname{rad} 1))=$ $\mu-\operatorname{rad}(v-\operatorname{rad} 1)=v-\operatorname{rad} 1 \cdot \mu-\operatorname{rad} 1$, hence the $\gamma-\operatorname{admissible}$ subset $\nu-\operatorname{rad} 1 \cdot \mu-\operatorname{rad} 1$ is linked with $\mu$. Analogously we can prove that such a subset is linked with $v$, hence by Lemma 1.3 it is linked with $v \vee \mu$. From Remark $1.2(v)$ it follows that $v$-rad $\cdot \mu$-rad $1 \supseteq(v \vee \mu)$-rad 1 . Moreover condition (iii) of Remark 1.2 implies $v$-rad $1 \cdot \mu$-rad $1 \subseteq(v \vee \mu)$-rad 1 hence $v-\operatorname{rad} 1 \cdot \mu-\operatorname{rad} 1=(v \vee \mu)-\operatorname{rad} 1$.

Lemma 1.6. Let $S=S\left(d, G_{i}, \gamma_{i}\right)$. If condition (*) holds, then $[l, \sigma \vee H]$ is a modular sublattice of $L(S)$.

Proof. Let $\rho, \lambda, \tau$ be congruencis on $S$ contained in $[l, \sigma \vee H]$ and such that $\rho \leqq \lambda$, $\rho \vee \tau=\lambda \vee \tau, \rho \wedge \tau=\lambda \wedge \tau$. We must prove that $\rho=\lambda$, that is $\operatorname{tr} \rho=\operatorname{tr} \lambda, A^{\rho}=A^{\lambda}$ (see Theorem B). By Theorem B, in $L(E)$ we have $\operatorname{tr} \rho \leqq \operatorname{tr} \lambda, \operatorname{tr} \rho \vee \operatorname{tr} \tau=\operatorname{tr} \lambda \vee \operatorname{tr} \tau$, $\operatorname{tr} \rho \wedge \operatorname{tr} \tau=\operatorname{tr} \lambda \wedge \operatorname{tr} \tau$; moreover, since $E$ is an $\omega$-chain, $L(E)$ is a modular lattice (see, e.g., [4, Theorem 2]) and $\operatorname{tr} \rho=\operatorname{tr} \lambda$. If we put $\nu=\operatorname{tr} \rho=\operatorname{tr} \lambda, \mu=\operatorname{tr} \tau$, then $v \vee \mu$ is a uniform congruence on $E(S)$ (being the trace of the congruence $\lambda \vee \tau$ of $S$ ) and $A^{\lambda} \cdot A^{\tau}$ is a $\gamma$-admissible subset of $G$ (see [2, p. 164)]. By condition (*), we have

$$
(v \vee \mu)-\operatorname{rad}\left(A^{\lambda} \cdot A^{\tau}\right)=A^{\lambda} \cdot A^{\tau} \cdot(v \vee \mu)-\operatorname{rad} 1 .
$$

Moreover $A^{\mathrm{\tau}}=\mu-\operatorname{rad} A^{\tau} \supseteq \mu-\operatorname{rad} 1$ and $A^{\lambda}=v-\operatorname{rad} A^{\lambda} \supseteq v-\operatorname{rad} 1$ so, by Lemma $1.5, A^{\lambda} \cdot A^{\tau} \supseteq$ $\mu$-rad $1 \cdot v-\operatorname{rad~} 1=(\mu \vee v)-\operatorname{rad} 1$. Hence (4) implies $(v \vee \mu)-\operatorname{rad}\left(A^{\lambda} \cdot A^{\tau}\right)=A^{\lambda} \cdot A^{\tau}$, whence, by Theorem B (iii), $A^{\lambda \vee \tau}=A^{\lambda} \cdot A^{\tau}$.

Analogously we prove that $A^{\rho \vee \tau}=A^{\rho} \cdot A^{\tau}$. Since $\lambda \vee \tau=\rho \vee \tau$, we have $A^{\rho} \cdot A^{\tau}=A^{\lambda} \cdot A^{\tau}$ and also $A^{\rho} \wedge A^{\tau}=A^{\rho \wedge \tau}=A^{\lambda \wedge \tau}=A^{\lambda} \wedge A^{\tau}$. From the modularity of the lattice of normal subgroups of a group (see also $\left[1\right.$, p. 464]), we have $A^{\rho}=A^{\lambda}$ and the lemma is proved.

Lemma 1.7. Let $S=S\left(d, G_{i}, \gamma_{i}\right)$ If $[1, \sigma \vee H]$ is a modular sublattice of $L(S)$, then $L(S)$ is modular.

Proof. Let $\lambda, \rho, \tau$ be congruences on $S$ such that $\rho \leqq \lambda, \rho \vee \tau=\lambda \vee \tau, \rho \wedge \tau=\lambda \wedge \tau$. We must prove that $\lambda=\rho$. By an argument similar to the one used in the proof of the preceding lemma, we have $\operatorname{tr} \rho=\operatorname{tr} \lambda$. Let $\operatorname{tr} \rho=\operatorname{tr} \lambda=v, \operatorname{tr} \tau=\mu$. If $v, \mu$ are both different from the universal relation $\omega_{E(S)}$ of $E(S)$, the congruences $\rho, \lambda, \tau$ belong to $[l, \sigma \vee H]$ (see $[2$, Remark p. 164]) and by the modularity of such sublattice we get $\lambda=\rho$. If, $v, \mu$ are both equal to $\omega_{E(S)}$, we have $\lambda=\rho$ because the lattice of group congruences on an inverse semigroup is modular (see [10, Corollary III.2.7]). Hence it remains to examine the following cases 
(i) $v=\omega_{E(S)}, \quad \mu \neq \omega_{E(S)}$

(ii) $\mu=\omega_{E(S)}, \quad v \neq \omega_{E(S)}$

We will use an argument analogous to the one used by Munn in the proof of Lemma 10 of [8].

Case (i). Since $\tau$ is not a group congruence, $\tau$ belongs to $[l, \sigma \vee H]$ hence $\tau=\tau \wedge(\sigma \vee H)$. So, since $\lambda \wedge(\sigma \vee H), \tau, \sigma \in[l, \sigma \vee H]$ and $\sigma \leqq \lambda \wedge(\sigma \vee H)(\lambda$ being a group congruence), it follows from the modularity of $[l, \sigma \vee H]$ that $(\lambda \wedge \tau) \vee \sigma=$ $(\lambda \wedge(\sigma \vee H) \wedge \tau) \vee \sigma=(\lambda \wedge(\sigma \vee H)) \wedge(\sigma \vee \tau)=\lambda \wedge((\sigma \vee H) \wedge(\sigma \vee \tau))=\lambda \wedge(\sigma \vee \tau)$. Analogously we can prove that $(\rho \wedge \tau) \vee \sigma=\rho \wedge(\sigma \vee \tau)$. From $\lambda \wedge \tau=\rho \wedge \tau$ and $\lambda \vee \tau=\rho \vee \tau$, we deduce

$$
\begin{aligned}
& \lambda \wedge(\sigma \vee \tau)=\rho \wedge(\sigma \vee \tau) \\
& \lambda \vee(\sigma \vee \tau)=\rho \vee(\sigma \vee \tau)
\end{aligned}
$$

The congruences $\lambda, \rho, \tau \vee \sigma$ are group congruences satisfying (5) and (6). Moreover $\rho \leqq \lambda$ and thus, from the modularity of the lattice of the group congruences of $S$, we have $\lambda=\rho$.

Case (ii). Since $\tau$ is a group congruence, $\tau \geqq \sigma$ and so $\tau \vee \sigma=\tau$, moreover $\tau$, $\lambda \vee \sigma$, $\sigma \vee H$ are group congruences, and $\lambda$ is not a group congruence, hence $\lambda \leqq \sigma \vee H$, so $\lambda \vee \sigma \leqq \sigma \vee H$. Since the lattice of the group congruences is modular, we have $(i \vee \tau) \wedge(\sigma \vee H)=((\lambda \vee \sigma) \vee \tau) \wedge(\sigma \vee H)=(\tau \wedge(\sigma \vee H)) \vee(\lambda \vee \sigma)=(\tau \wedge(\sigma \vee H)) \vee \lambda$. Analogously we can deduce that $(\rho \vee \tau) \wedge(\sigma \vee H)=(\tau \wedge(\sigma \vee H)) \vee \rho$. Thus, from $i \vee \tau=\rho \vee \tau$ it follows

$$
(\tau \wedge(\sigma \vee H)) \vee \lambda=(\tau \wedge(\sigma \vee H)) \vee \rho
$$

Moreover from $\lambda \wedge \tau=\rho \wedge \tau$ it follows

$$
(\tau \wedge(\sigma \vee H)) \wedge \lambda=(\tau \wedge(\sigma \vee H)) \wedge \rho
$$

The congruences $\lambda, \rho, \tau \wedge(\sigma \vee H)$ belong to the modular sublattice $[1, \sigma \vee H]$ of $L(S)$ and satisfy (7) and (8), moreover, $\rho \leqq \lambda$, hence we have again $\rho=\lambda$ and the lemma is proved.

Now we are able to state the following characterization of simple regular $\omega$ semigroups whose lattice of congruences is modular.

Theorem 1.8. Let $S=S\left(d, G_{i}, \gamma_{i}\right)$. L $(S)$ is modular is and only if, for every $\gamma$-admissible subset $A=A_{0} \times \cdots \times A_{d-1}$ of $G$, one of the following conditions holds 
(i) $A \cdot \mu-\operatorname{rad} 1=\mu-\operatorname{rad} A$ for every uniform congruence $\mu$ on $E(S)$,

(ii) $A_{0} \gamma_{d-1}^{-1}=A_{d-1} \cdot \operatorname{ker} \gamma_{d-1}$ and $A_{k+1} \gamma_{k}^{-1}=A_{k} \cdot \operatorname{ker} \gamma_{k}$ for every $k$ with $0 \leqq k \leqq d-2$.

Proof. From previous lemmas we can deduce that the congruences lattice of $S$ is modular if and only if condition (i) holds.

Now we prove that (i) implies (ii). For $0 \leqq k \leqq d-1$, let $\mu_{k}$ the congruence on $E$ defined by

$$
\begin{aligned}
\mu_{k}=\left\{\left(e_{i}^{n}, e_{j}^{m}\right) \mid i=j, n=m \text { if } j \neq k ; \text { or } i=j-1, n=m \text { if } j=k \neq 0 ;\right. \\
\\
\quad \text { or } i=d-1, n=m-1 \text { if } j=k=0\},
\end{aligned}
$$

and let $A=A_{0} \times \cdots \times A_{d-1}$ be a $\gamma$-admissible subset of $G$, it immediately follows that $\mu_{k}-\operatorname{rad} A=A_{0} \times \cdots \times A_{k-2} \times A_{k} \gamma_{k-1}^{-1} \times A_{k} \times \cdots \times A_{d-1}$ for every $0<k \leqq d-1$ and $\mu_{0}-\operatorname{rad} A=A_{0} \times \cdots \times A_{d-2} \times A_{0} \gamma_{d-1}^{-1}$. So from (i) we deduce condition (ii).

Now we will prove that (ii) implies (i). From $[2,2]$, and from definition of $\mu$-rad $A$ it follows that it is enough to prove that

$$
A_{j(\bmod d)} \alpha_{i, j}^{-1}=A_{i(\bmod d)} \cdot \operatorname{ker} \alpha_{i, j}
$$

for every positive integers $i, j$ with $i<j$. Denote by $\bar{j}$ and $\bar{i}$ the integers $j(\bmod d)$ and $i$ $(\bmod d)$ respectively, and distinguish two cases:

Case 1. Let $j-i<d$. Suppose $\bar{i}<\bar{j}$. The subset

$$
A_{0} \times \cdots \times A_{i} \times A_{j} \alpha_{i+1, j}^{-1} \times A_{j} \alpha_{i+2, j}^{-1} \times \cdots \times A_{j} \alpha_{j-1, j}^{-1} \times A_{j} \times \cdots \times A_{d-1}
$$

is a $\gamma$-admissible subset of $G$. hence, by (ii), we have $A_{\bar{j}} \alpha_{\bar{i}+1, j}^{-1} \gamma_{\bar{i}}^{-1}=A_{\bar{i}} \cdot \operatorname{ker} \gamma_{\bar{i}} \subseteq A_{\bar{i}} \operatorname{ker} \alpha_{\bar{i}, \bar{j}}$; since obviously $A_{\bar{j}} \alpha_{i+1, j}^{-1} \gamma_{\bar{i}}^{-1} \supseteq A_{\bar{i}} \operatorname{ker} \alpha_{\bar{i}, j}$ the statement follows. If $\bar{j}<\bar{i}$, we consider the $\gamma$-admissible subset

$$
A_{0} \alpha_{0, j}^{-\frac{1}{j}} \times \cdots \times A_{j} \alpha_{j-1, j}^{-1} \times A_{j} \times \cdots \times A_{i} \times A_{j} \alpha_{i+1, j}^{-1} \times \cdots \times A_{j} \alpha_{d-1, j}^{-1}
$$

and analogously we deduce (9).

Case 2. Let $j-i \geqq d$, suppose $\bar{i}<\bar{j}$ then $\alpha_{i, j}=\alpha_{\bar{i}, j} \alpha_{j}^{n}$ for some positive integer $n$. We establish (9) by induction on $n$, the case $n=0$ being proved by Case 1 . We consider the $\gamma$-admissible subset

$$
\begin{aligned}
& A_{j}\left(\alpha_{0 . j} \alpha_{j}^{n-1}\right)^{-1} \times \cdots \times A_{j}\left(\alpha_{i, j} \alpha_{j}^{n-1}\right)^{-1} \times A_{j}\left(\alpha_{\bar{i}+1, j} \alpha_{j}^{n}\right)^{-1} \times \cdots \\
& \quad \times A_{j}\left(\alpha_{j}^{n}\right)^{-1} \times A_{j}\left(\alpha_{j+1 . j} \alpha^{\alpha} \alpha_{j}^{n-1}\right)^{-1} \times \cdots \times A_{j}\left(\alpha_{d-1, j+d} \alpha_{j}^{n-1}\right)^{-1},
\end{aligned}
$$

by (ii) and by the induction hypothesis we have 


$$
\begin{aligned}
A_{\bar{j}}\left(\alpha_{\bar{i}+1, j} \alpha_{\bar{j}}^{n}\right)^{-1} \gamma_{i}^{-1} & =A_{\bar{j}}\left(\alpha_{\bar{i}, j} \alpha_{j}^{n-1}\right)^{-1} \cdot \operatorname{ker} \gamma_{i}=A_{j} \cdot \operatorname{ker} \alpha_{\bar{i}, j} \alpha_{\bar{j}}^{n-1} \cdot \operatorname{ker} \gamma_{i} \\
& =A_{\bar{j}}=A_{\bar{j}} \cdot \operatorname{ker} \alpha_{\bar{i}, \bar{j}} \alpha_{\bar{j}}^{n-1} \subseteq A_{\bar{j}} \cdot \operatorname{ker} \alpha_{\bar{i}, j} \alpha_{j}^{n} .
\end{aligned}
$$

Since $A_{\bar{j}} \cdot \operatorname{ker} \alpha_{\bar{i}, j} \alpha_{j}^{n} \subseteq A_{\bar{j}}\left(\alpha_{\bar{i}+1, j} \alpha_{\bar{j}}^{n}\right)^{-1}$, we have condition (9). Analogously we proceed when $\bar{i} \geqq \bar{j}$,

Remark 1.9. If $S$ is a bisimple $\omega$-semigroup then $S$ is a simple regular $\omega$-semigroup with $d=1$, hence from Theorem 1.8 we reobtain the Munn's result (see [8, 2]). In fact, the congruences on a bisimple $\omega$-semigroup are either idempotent separating or group congruences (see $[8,1]$ ), the $\gamma$-admissible subsets coincide with the $\alpha$-admissible normal subgroups of Munn and $\omega_{E}-\operatorname{rad} A=\operatorname{rad} A$.

From Theorem 1.8, we can also deduce Theorem 4.1 of [1].

Remark 1.10. For every positive integer $d$ there exists a simple regular $\omega$-semigroup $S=S\left(d, G_{i}, \gamma_{i}\right)$ whose lattice of congruences is modular. In fact if $\gamma_{i}$ is trivial for every $i=0,1, \ldots, d-1$, then condition (ii) of Theorem 1.8 holds. Furthermore we prove that if a simple regular $\omega$-semigroup $S$ satisfies (ii) of Theorem 1.8 and there exists a $k$ such that $\gamma_{k}$ is trivial, then $\gamma_{i}$ is trivial for every $i$. Let us consider the $\gamma$-admissible subset

$$
A_{k}=\left\{e_{0}\right\} \times \cdots \times\left\{e_{k-1}\right\} \times G_{k} \times\left\{e_{k+1}\right\} \times \cdots \times\left\{e_{d-1}\right\}
$$

from condition (ii) of Theorem 1.8 we deduce $\operatorname{ker} \gamma_{k-1}=G_{k-1}$, so, $\gamma_{k-1}$ is trivial. Repeating this argument, we can prove that $\gamma_{i}$ is trivial for every $i=0,1, \ldots, d-1$ (we remark that triviality of $\gamma_{0}$ implies triviality of $\gamma_{d-1}$ ).

From the previous remark we deduce that, differently from what happens in the bisimple $\omega$-semigroup (see [8, Corollaries 2 and 5]), the finiteness or the simplicity of $G_{i}$ do not imply, in general, the modularity of $L(S)$. We also remark that it is possible to construct $S=S\left(d, G_{i}, \gamma_{i}\right)$ with a modular lattice of congruences and $\gamma_{i}$ non trivial. We can for example take $d=2, G_{0}=S_{3}, G_{1}=\left\{e_{1}, g\right\}, \gamma_{0}$ such that $x \gamma_{0}=e_{1}$ if $x$ belongs to the alternating subgroup of $G_{0}$, otherwise $x \gamma_{0}=g, \gamma_{1}$ such that $e_{1} \gamma_{1}=i, g \gamma_{1}=(12)$, where $i$ denotes the identity of $S_{3}$.

Finally we remark that the modularity of $L(S)$ does not imply that the congruences of $L(S)$ are permutable (see [3]).

\section{The general case}

First we recall that, for a regular $\omega$-semigroup, Munn in [7] proved the following result: 
Theorem C. Let $S$ be a regular $\omega$-semigroup.

(i) If $S$ has no kernel, then it is the union of an $\omega$-chain of groups

(ii) If the kernel of $S$ coincides with $S$, then $S$ is a simple regular $\omega$-semigroup

(iii) If $S$ has a proper kernel, then $S$ is a (retract) ideal extension of a simple regular $\omega$ semigroup $K$ by a semigroup $H^{0}$, where $H$ is a finite chain of groups and $H^{0}$ is obtained from $H$ by adjoining a zero. Moreover this extension is determined by means of $a$ homomorphism of $H$ into the group of units of $K$.

The characterization of semigroups of type (i) whose lattice of congruence is modular can be easily deduced from the following:

Theorem D. (See [4, Theorem 3]). Let $S$ be a semilattice of groups and $E$ its semilattice of idempotents. Then $L(S)$ is modular if and only if $E$ is a tree and $S$ has trivial multiplication.

In fact, from Theorem D immediately follows:

Theorem 2.1. Let $S=\left[\Omega, H_{j}, \phi_{j}\right]$ be a regular $\omega$-semigroup without kernel. Then $L(S)$ is modular if and only if $\phi_{j}$ are trivial for every $j \in \mathbb{N}$. $\left(S=\left[\Omega, H_{j}, \phi_{j}\right]\right.$ indicates the Clifford representation of the $\omega$-chain $\Omega$ of the groups $H_{j}(j \in \mathbb{N})$ with connecting homomorphisms $\phi_{j}=\phi_{j, j+1}$ )

Theorem 1.8 gives the characterization of semigroups of type (ii) whose lattice of congruences is modular. To achieve the aim of the paper, it remains to examine the semigroups of type (iii). Such a semigroup $S$ is the disjoint union of a finite chain of groups $H$ and of a simple regular $\omega$-semigroup $K$ which is an ideal of $S$.

For $H$ we use the short Clifford representation $\left[n, H_{j}, \phi_{j}\right]$, where $n$ is the length of the chain, $j=0, \ldots, n-1, \phi_{j}=\phi_{j, j+1}$ for $0 \leqq j \leqq n-2$, and use for $K$ the notation $K=$ $K\left(d, K_{i}, \psi_{i}\right)$. Moreover, since the group of units of $K$ is $\left\{\left(0, k_{0}, 0\right) \mid k_{0} \in K_{0}\right\}$, the homomorphism $\phi$ which induces the retract extension of $K$ by $H^{0}$ can be constructed by means of a homomorphism $\phi_{n-1}: H_{n-1} \rightarrow K_{0}$ in the following way

$\phi: h_{n-1} \rightarrow\left(0, h_{n-1} \phi_{n-1}, 0\right)$ for every $h_{n-1} \in H_{n-1}$

$\left.\phi: h_{i} \rightarrow\left(0,\left(\left(h_{i} \phi_{i}\right) \phi_{i+1}\right) \cdots\right) \phi_{n-1}, 0\right)$ for every $h_{i} \in H_{i}: 0 \leqq i \leqq n-2$.

So $S$ can be represented by the following diagram of homomorphisms

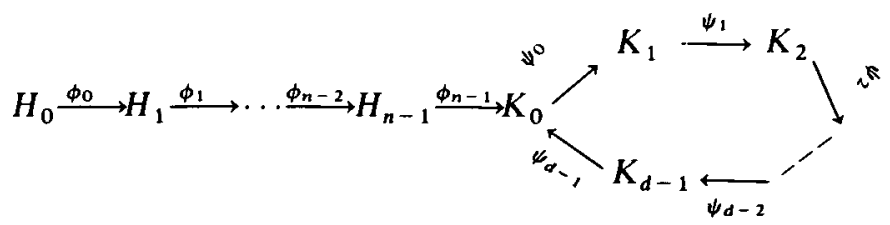

We will denote $S$ by the notation $S=S\left(\left[n, H_{j}, \phi_{j}\right] ; K\left(d, K_{i}, \psi_{i}\right) ; \phi_{n-1}\right)$, or shortly, 
$S=S\left(H ; K ; \phi_{n-1}\right)$. Moreover we will indicate the idempotents of $S$ by: $f_{i}$ is the identity of $H_{j}(j=0, \ldots, n-1), e_{i}^{m}=\left(m, e_{i}, m\right)\left(m \in \mathbb{N} ; i=0, \ldots, d-1 ; e_{i}\right.$ the identity of $\left.K_{i}\right)$ are the idempotents of $K$. Therefore

$$
f_{0}>f_{1}>\cdots>f_{n-1}>e_{0}^{0}>e_{1}^{0}>\cdots>e_{d-1}^{0}>e_{0}^{1}>\cdots
$$

Lemma 2.2. Let $S=S\left(H ; K ; \phi_{n-1}\right)$ and let $L(S)$ be modular. Then

(a) $L(H)$ is modular

(b) $L(K)$ is modular

(c) $\phi_{n-1}$ is trivial.

Proof. First we prove (a). Let $\rho_{K}$ be the Rees congruence of $S$ modulo $K$. Since $L(S)$ is modular, $L\left(S / \rho_{K}\right)$, that is $L\left(H^{0}\right)$, is modular whence also $L(H)$ is modular (see, e.g., [4, Corollary of Theorem 1 and proof of Theorem 4]).

Now we prove (b). For every congruence $\rho^{\prime}$ on $K$, denote by $\rho$ the congruence defined by $a \rho b$ if and only if either $a, b \in K$ and $a \rho^{\prime} b$ or $a=b$. It is immediate that $\rho$ is a congruence on $S$ and that the mapping $\rho^{\prime} \rightarrow \rho$ is an injective lattice homomorphism from $L(K)$ into $L(S)$. Consequently, since $L(S)$ is modular, so is $L(K)$.

Finally we prove (c). Let $\rho, \lambda, \tau$, be relations on $S$ respectively defined by $a \rho b$ if and only if either $a=b$ or $a, b \in K$, or $a, b \in H_{n-1}$ and $a \phi_{n-1}=b \phi_{n-1}, a \lambda b$ if and only if either $a=b$, or $a, b \in K$, or $a, b \in H_{n-1}, a \tau b$ if and only if either $a=b$, or $a, b \in H_{n-1}$ and $a \phi_{n-1}=b \phi_{n-1}$, or $a \in K, b \in H_{n-1}$ and $b \phi_{n-1}=a$, or $b \in K, a \in H_{n-1}$ and $a \phi_{n-1}=b$.

It is straightforward to verify that $\rho, \lambda, \tau$ are congruences on $S$ such that $\rho \leqq \lambda$, $\rho \vee \tau=\lambda \vee \tau, \rho \wedge \tau=\lambda \wedge \tau$. Since $L(S)$ is modular, we have $\rho=\lambda$, hence $a \phi_{n-1}=b \phi_{n-1}$ for every $a, b \in H_{n-1}$, thus $\phi_{n-1}$ is trivial.

Lemma 2.3. Let $S=\left(H ; K ; \phi_{n-1}\right)$ with $\phi_{n-1}$ trivial. Then for all congruences $\rho, \tau$ on $S$ we have

(a) $(\rho \vee \tau)_{\mid K}=\rho_{\mid K} \vee \tau_{\mid K}$

(b) $(\rho \vee \tau)_{\mid H}=\rho_{\mid H} \vee \tau_{\mid H}$

(c) $(\rho \wedge \tau)_{\mid K}=\rho_{\mid K} \wedge \tau_{\mid K}$

(d) $(\rho \wedge \tau)_{\mid H}=\rho_{\mid H} \wedge \tau_{\mid H}$

(e) $\operatorname{ker} \rho=\operatorname{ker} \rho_{\mid H} \cup \operatorname{ker} \rho_{\mid K}$

Proof. First we prove condition (a). We immediately have that $(\rho \vee \tau)_{\mid K} \geqq \rho_{\mid K} \vee \tau_{\mid K}$, hence we verify that

$$
(\rho \vee \tau)_{\mid K} \leqq \rho_{\mid K} \vee \tau_{\mid K}
$$


Let $a, b \in K$ such that $a(\rho \vee \tau)_{1 K} b$. Then there exist $x_{1}, x_{2}, \ldots, x_{r} \in S$ such that $a \rho x_{1}$, $x_{1} \tau x_{2}, \ldots, x_{r} \tau b$. Hence $a=a e_{0}^{0} \rho x_{1} e_{0}^{0}, x_{1} e_{0}^{0} \tau x_{2} e_{0}^{0}, \ldots, x_{r} e_{0}^{0} \tau b e_{0}^{0}=b$. All the elements $x_{i} e_{0}^{0}$ belong to $K$, thus $a\left(\rho_{\mid K} \vee \tau_{\mid K}\right) b$.

Now we prove condition (b). It is enough to prove that $(\rho \vee \tau)_{\mid H} \leqq \rho_{\mid H} \vee \tau_{\mid H}$. Let $a, b \in H$ with $a(\rho \vee \tau)_{\mid H} b$. Then there exist $x_{1}, x_{2}, \ldots, x_{r} \in S$ such that

$$
a \rho x_{1}, x_{1} \tau x_{2}, \ldots, x_{r} \tau b
$$

If all the elements $x_{1}, x_{2}, \ldots, x_{r} \in S$ belong to $H$, then $\left(\rho_{\mid H} \vee \tau_{\mid H}\right) b$. Suppose, on the contrary, that there are in (10) some elements of $K$, i.e. that (10) contains sequences of the type

$$
h \sigma_{1} k_{1}, k_{1} \sigma_{2} k_{2}, \ldots, k_{s-1} \sigma_{s} h^{\prime}
$$

where $h, h^{\prime} \in H, k_{1}, k_{2}, \ldots, k_{s-1} \in K$ and, for every $i=1, \ldots, s$, either $\sigma_{i}=\rho$ or $\sigma_{i}=\tau$.

Suppose, without loss of generality, that $h \in H_{i}$ and $h^{\prime} \in H_{j}, i, j \in\{0,1, \ldots, n-1\}, i \geqq j$. Thus, since $\phi_{n-1}$ is trivial, we have, using the first pair of (11), $h^{-1} h \sigma_{1} h^{-1} k_{1}$ hence $f_{i} \sigma_{1} k_{1}$ and $f_{i} \sigma_{1} h$, using the last, $k_{s-1}\left(h^{\prime}\right)^{-1} \sigma_{s} h^{\prime}\left(h^{\prime}\right)^{-1}$ hence $k_{s-1} \sigma_{s} f_{j}, k_{s-1} f_{i} \sigma_{s} f_{j} f_{i}$, $k_{s-1} \sigma_{s} f_{i}$ and $f_{i} \sigma_{s} h^{\prime}$, hence we can replace sequence (11) with the sequence $h \sigma_{1} f_{i}$, $f_{i} \sigma_{s} h^{\prime}$ whose elements are in $H$, whence $a\left(\rho_{\mid H} \vee \tau_{\mid H}\right) b$.

The proofs of (c) and (d) are trivial and obviously hold if $H$ and $K$ are replaced by an arbitrary subsemigroup $T$ and $S$.

Finally we must prove (e). It is obvious that $\operatorname{ker} \rho \supseteq \operatorname{ker} \rho_{\mid H} \cup \operatorname{ker} \rho_{\mid K}$. Suppose that $\operatorname{ker} \rho \supset \operatorname{ker} \rho_{\mid H} \cup \operatorname{ker} \rho_{\mid K}$. Two cases are possible.

Case 1: there exists $x \in K$ such that $x \rho f_{j}$ for some $j=0,1, \ldots, n-1$ and $x \notin \operatorname{ker} \rho_{\mid K}$.

Case 2: there exists $y \in H$ such that $y \rho e_{i}^{m}$ for some $i=0,1, \ldots, d-1, m \in \mathbb{N}$, $y \notin \operatorname{ker} \rho_{\mid H}$.

In the first case from $x \rho f_{j}$ it follows $x^{-1} x \rho x^{-1} f_{j}$ and, since $f_{j}$ is the identify of $H_{j}$ and $x^{-1} x$ is an idempotent $e_{i}^{m}$ of $K$, we get $e_{i}^{m} \rho x^{-1} e_{0}^{0}=x^{-1}$; hence $x^{-1} \in \operatorname{ker} \rho_{\mid K}$ and $x \in \operatorname{ker} \rho_{\mid K}$, a contradiction.

In the second case from $y \rho e_{i}^{m}$ it follows $y^{-1} y \rho y^{-1} e_{i}^{m}$ and, since $y^{-1} y$ is the identity of the group $H_{j}$ and $\phi_{n-1}$ is trivial, $f_{j} \rho e_{0}^{0} e_{i}^{m}, f_{j} \rho e_{i}^{m}, f_{j} \rho y$, hence we obtain the contradiction $y \in \operatorname{ker} \rho_{\mid H}$. Thus $\operatorname{ker} \rho=\operatorname{ker} \rho_{\mid H} \cup \operatorname{ker} \rho_{\mid K}$.

Lemma 2.4. Let $S=S\left(H ; K ; \phi_{n-1}\right)$ and suppose that the following conditions hold

(a) $L(H)$ is modular

(b) $L(K)$ is modular

(c) $\phi_{n-1}$ is trivial

Then $L(S)$ is modular.

Proof. Let $\rho, \lambda, \tau$ be congruences on $S$ such that $\rho \leqq \lambda, \rho \vee \tau=\lambda \vee \tau, \rho \wedge \tau=\lambda \wedge \tau$. 
Since the mapping $\rho \rightarrow \operatorname{tr} \rho$ is a lattice homomorphism of $L(S)$ into $L(E)$ (see e.g. [10, Theorem III.2.5]) and $L(E)$ is modular, $E$ being an $\omega$-chain (see e.g. [4, Theorem 2]), we have $\operatorname{tr} \rho=\operatorname{tr} \lambda$. On the other hand, by Lemma 2.3 , we have

$$
\begin{aligned}
& (\lambda \vee \tau)_{\mid K}=\lambda_{\mid K} \vee \tau_{\mid K} \\
& (\rho \vee \tau)_{\mid K}=\rho_{\mid K} \vee \tau_{\mid K} \\
& (\lambda \wedge \tau)_{\mid K}=\lambda_{\mid K} \wedge \tau_{\mid K} \\
& (\rho \wedge \tau)_{\mid K}=\rho_{\mid K} \wedge \tau_{\mid K}
\end{aligned}
$$

Since $\rho_{\mid K} \leqq \lambda_{\mid K}$ it follows from the modularity of $L(K)$ that $\rho_{\mid K}=\lambda_{\mid K}$. Analogously we can prove $\rho_{\mid H}=\lambda_{\mid H}$. From condition (e) of Lemma 2.3 , we deduce also $\operatorname{ker} \lambda=\operatorname{ker} \lambda_{\mid K} \cup$ $\operatorname{ker} \lambda_{\mid H}=\operatorname{ker} \rho_{\mid K} \cup \operatorname{ker} \rho_{\mid H}=\operatorname{ker} \rho$. Thus, since $\operatorname{tr} \rho=\operatorname{tr} \lambda$ and $\operatorname{ker} \lambda=\operatorname{ker} \rho$, we have $\rho=\lambda$ (see, for instance [10, Theorem III.1.5]), hence $L(S)$ is modular.

The above lemmas, Theorem $\mathrm{D}$ and Theorem 1.8, give us the following theorem which completely characterizes the regular $\omega$-semigroups whose lattice of congruences is modular.

Theorem 2.5. Let $S=S\left(\left[n, H_{j}, \phi_{j}\right] ; K\left(d, K_{i}, \psi_{i}\right) ; \phi_{n-1}\right)$ be a regular $\omega$-semigroup with proper kernel. Then $L(S)$ is modular if and only if the following hold.

(a) $\phi_{0}, \phi_{1}, \ldots, \phi_{n-1}$ are trivial,

(b) $A_{0} \psi_{d-1}^{-1}=A_{d-1} \cdot \operatorname{ker} \psi_{d-1}$ and $A_{k+1} \psi_{k}^{-1}=A_{k} \cdot \operatorname{ker} \psi_{k}(0<k \leqq d-1)$ for every $\psi$ admissible subset $A=A_{0} \times \cdots \times A_{d-1}$ of $K_{0} \times \cdots \times K_{d-1}$.

Remark 2.6. We remark that Theorems 2.1 and 2.5 could be deduced either from Theorem 1.21 or from Theorem 5.2 of [6]. Since the proofs of these theorems have not been published, we have given here a direct proof of our result.

After the authors sent the paper to this journal, they knew that M. Petrick proved in "Congruences on strong semilattices of regular simple semigroups", Semigroup Forum 37 (1988), 167-199, a theorem from which our Theorem 2.5 can be immediately deduced.

\section{REFERENCES}

1. G. R. BAIRD, On a sublattice of the lattice of congruences on a simple regular $\omega$-semigroup, J. Austral. Math. Soc. 13 (1972), 461-471.

2. G. R. Baird, Congruences on simple regular $\omega$-semigroups, J. Austral. Math. Soc. 14 (1972). 155-167.

3. C. Bonzini and A. Cherubini, Permutable regular $\omega$-semigroups, Boll. Un. Mat. Lial. (7) 2-B (1988), 719-728. 
4. J. B. Fountaln and P. Lockley, Semilattices of groups with distributive congruence lattices, Semigroup Forum 14 (1977), 81-91.

5. B. P. Kocis, The structure of inverse ideally simple $\omega$-semigroups, Vestnik Leningrad Univ. 23(7) (1968), 41-50.

6. H. Мıтsсн, Semigroups and their lattice of congruences, Semigroup Forum 26 (1983), 1-63.

7. W. D. MunN, Regular $\omega$-semigroups, Glasgow Math. J. 9 (1968), 46-66.

8. W. D. Munn, The lattice of congruences on a bisimple $\omega$-semigroup, Proc. Roy. Soc. Edinburgh Sect. 67 (1965/67), 175-184.

9. M. Petrich, M. Congruence on simple $\omega$-semigroups, Glasgow Math. J. 20, (1979), 87-101.

10. M. Petrich, Inverse Semigroups (Wiley \& Sons 1984).

Dipartimento Di Matematica

UNIVERSITÁ

VIA SALDINI, 50

20133, Milano

ITALY
Dipartimento Di Matematica Politecnico

Piazza L. Da Vinci, 32

20133, Milano

ITALY 